\title{
Aortic dissection: A little help from our friends
}

Joseph S. Coselli, MD

\footnotetext{
From the Division of Cardiothoracic Surgery, Michael E. DeBakey Department of Surgery, Baylor College of Medicine, Houston, Tex; Section of Adult Cardiac Surgery, Department of Cardiovascular Surgery, Texas Heart Institute, Houston, Tex; and CHI St Luke's Health-Baylor St Luke's Medical Center, Houston, Tex.

Disclosures: Author has nothing to disclose with regard to commercial support.

Received for publication Nov 30, 2017; accepted for publication Dec 1, 2017; available ahead of print Jan 20, 2018.

Address for reprints: Joseph S. Coselli, MD, One Baylor Plaza, BCM 390, Houston, TX 77030 (E-mail: jcoselli@ bcm.edu).

J Thorac Cardiovasc Surg 2018; 155:2249-50

$0022-5223 / \$ 36.00$

Copyright (C) 2017 by The American Association for Thoracic Surgery

https://doi.org/10.1016/j.jtcvs.2017.12.020
}

Determining the incidence of virtually any disease is crucial. All societies must proportionately allocate appropriate resources and plan accordingly for specific management challenges based on their health care system. This allocation is particularly true for patients with thoracic aortic dissection or aneurysm. From a 12-year study period, McClure and colleagues ${ }^{1}$ present insight into the incidence and early management of thoracic aortic dissection and aneurysms for the 13.5 million residents of Ontario, Canada, which is the most populated province in Canada. For their analysis, the authors have accessed data from multiple administrative databases and networks that contain both provincial and national data. This collective information has a unique value: it is from a relatively stable population and is comprehensive, including data from hospitals, outpatient clinics, and emergency department visits. The authors find that there is an increased incidence of thoracic aortic dissection and aneurysm for residents of Ontario and speculate the increase may be due to improved identification and enhanced awareness. The data and findings are very likely representative of most, if not all, the Western world.

Overall, they found an increased incidence in aortic dissection, which was driven almost entirely by a $4 \%$ increase in Stanford type A aortic dissections; however, there was no increase in type B aortic dissections. McClure and colleagues ${ }^{1}$ are astutely aware that an acute type A aortic dissection nearly universally necessitates emergent surgery to restore blood into the true lumen and to overcome related scenarios of life-threatening aortic valvular regurgitation, pericardial tamponade, and aortic rupture. They were justifiably interested to note that only $53 \%$ of patients who were diagnosed with type A aortic dissection actually had surgery; operative mortality was $20.6 \%$, a rate that did not substantially change over the 12-year period of analysis. However, overall hospital mortality (which included nonoperative medical management, surgery, and endovascular repair) for type A aortic dissection did decrease from $65 \%$ in 2002 to $48 \%$ in 2012. over time.

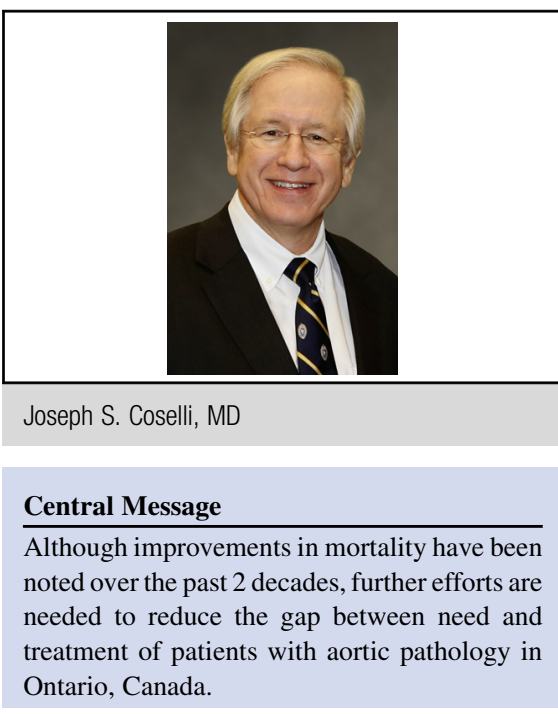

See Article page 2254.

Regarding type B aortic dissections, $83 \%$ of patients were treated with medical management alone, $10 \%$ underwent open surgery, and 7\% underwent endovascular repair; operative mortality for type B dissection was 5.6\% with no change over time. For patients with thoracic aortic aneurysm, 44\% were treated with medical management alone, 53\% underwent open surgery, and 3\% underwent endovascular repair. In addition, at 3 years, survival for patients with either aortic dissection or aneurysm improved

This study from McClure and colleagues ${ }^{1}$ is essential and highlights some harsh truths regarding unequal access to care and underuse of emerging technology by cardiac surgeons. Notably, it is difficult to understand why only one half of patients with a type A aortic dissection undergo surgery. The authors speculate this may be due to the challenge of definitively diagnosing aortic dissection and the complexities of transporting patients to suitable specialty centers. Additionally concerning was the finding that significantly fewer women underwent surgery to treat type A dissection, consequently resulting in much greater rates of overall mortality. Regarding the nearly 10,000 patients with aneurysm, although the ability to delineate the types of aneurysm was somewhat limited, open thoracoabdominal aortic aneurysm repair was undertaken in only 296 , with an operative mortality rate of $16 \%$, which was consistent over time. Although the morality rate is not unduly excessive, the fact that there has been no 
improvement over a 12-year period is both disconcerting and a marker to focus attention.

Finally, the authors report that only $8 \%$ of endovascular aortic repairs had a cardiac surgeon as the primary clinician. The obvious conclusion may be that there is a significant need for integrating more endovascular skills into the cardiac surgeon training program and a need for greater exposure to endovascular techniques in general. Possibly, the growing experience with catheter skills related to transcatheter aortic valve replacement may aid the development of skills necessary for endovascular aortic repair.

On a positive note, the authors point out that when the time from diagnosis to definitive hospitalization was reduced, fewer patients died. The authors note a key trend toward the development of specific programs with aortic repair experience and expertise. Within the province, 11 centers performed a majority of open surgery and endovascular repair for aneurysms and dissections. There are obvious benefits of improved diagnostic capabilities and rapid transfer to experienced aortic centers.

The authors provide a fascinating population-based study that provides a wealth of information for analysis and comparison. I believe their single-most salient and universal point is that despite the great progress in the 2 decades regarding the treatment of aortic disease, there is a large gap between need and treatment. If we are to serve our patients at a level truly commensurate with need, then a great deal more needs to be done to close the gap, from providing equitable access to emergent care to ensuring surveillance of patients with a known thoracic aneurysm.

\section{Reference}

1. McClure RS, Brogly SB, Lajkosz K, Payne D, Hall SF, Johnson AP. Epidemiology and management of thoracic aortic dissections and thoracic aortic aneurysms in Ontario, Canada-A population-based study. J Thorac Cardiovasc Surg. 2018; 155:2254-64.e4. 